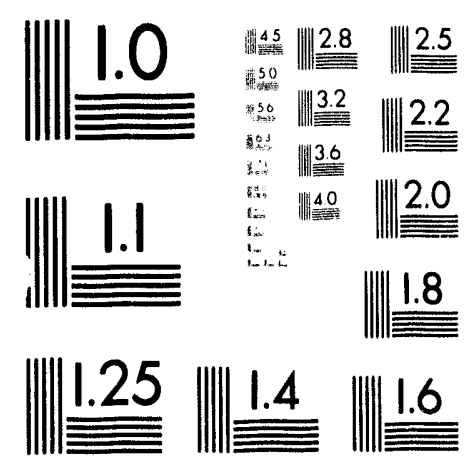



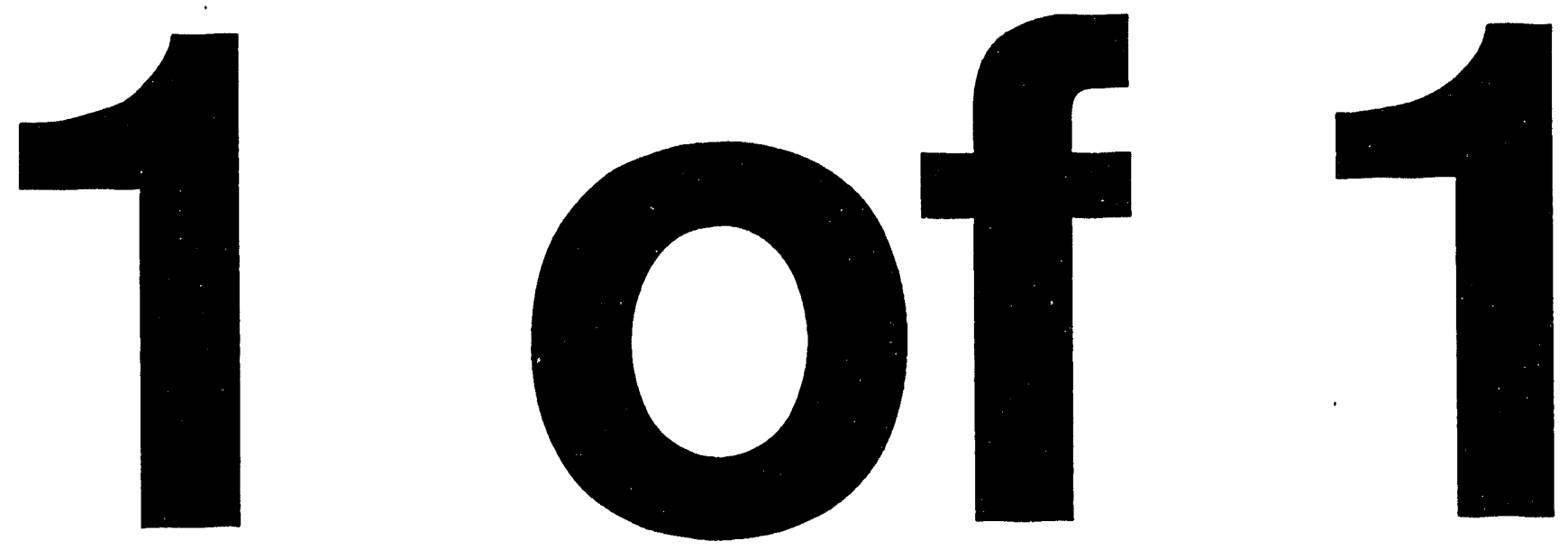

$-\longrightarrow$ 


\title{
1993 Annual Report for the Phaedrus-T RF Current Drive Experiments
}

\author{
Phaedrus-T Group \\ University of Wisconsin - Madison \\ Noah Hershkowitz - Principal Investigator.
}

\section{Introduction and Summary:}

After a series of antenna modifications and a program of optimizing our Boronization procedures, we have succeeded in coupling $300 \mathrm{~kW}$ of $\mathrm{RF}$ power to the plasma. Thomson Scattering shows a $20-60 \%$ increase in core $\mathrm{T}_{\mathrm{e}}$, and constitutes experimental evidence that the waves are interacting with the electron population. Beam Emission Spectroscopy (BES) data show that the power is deposited in the core and at the edge as predicted by theoretical modeling. Ninety degree phasing of the antenna caused loop voltage drops of $15-25 \%$, which can be interpreted as an increase $5 \mathrm{kA}$ of toroidal plasma current for co-injection phasing versus counter injection phasing. Biased $\mathrm{H}$-modes have been created with a biased electrode. These plasmas have a steeper edge density gradient and reduced edge fluctuations than our normal limiter plasmas and a $D_{\alpha}$ emission drop at the limiter. Radial profiles of soft X-ray line emission have been measured by the Johns Hopkins group and significantly aided in our understanding of impurity generation with $\mathrm{RF}$ and in the biased $\mathrm{H}$-mode. Initial reflectometry data shows the presence of RF density fluctuations in the plasma. Different boronization techniques have been tried leading to a higher boron content in the deposited layer and reduced wall recycling. In this report, we outline the series of experiments that we have performed in the last year that led us to our present encouraging results.

\section{RF Heating, Current Drive Experiments and Associated BES Results:}

Many different antennas have been installed in Phaedrus-T, and with our current version and improved boronization we are observing substantial loop voltage drops, electron heating, and RF power deposition in the core of the tokamak plasma. The final antenna modifications to a 2 strap version with no crossover straps on the RF feeds have dramatically improved its behavior, and enabled us to observe loop voltage drops of 20$30 \%$ for $0 / \pi$ phasing, and suppress the RF power induced fueling from gas reflux and impurity generation.

A low power experiment was carried out by frequency sweeping the two strap antenna during a tokalmak discharge from $1-20 \mathrm{MHz}$ at a power of 500 watts. We found the Alfven wave signature with probes and rapidly surveyed a large range of tokamak operating regimes. We identified the most promising operating parameters for the full power Alfven RF current drive experiments including the appropriate magnetic field, RF frequency and plasma density.

The 2 strap antemna was retuned to 7 MHz, and we succeeded in coupling $300 \mathrm{~kW}$ to the plasma. For an antenna phasing of $\mid(0, \pi \mid$, we measured loop voltage drops of 20 $30 \%$ along with Thomson Scattering meatsurements of a 20-60\% increase of the electron temperature, from 35()$\pm 5() \mathrm{eV}$ to 55()$\pm 5() \mathrm{eV}$ in the core. The drop in loop voltage is not an artifact of a change in density, and does not depend on impurity (SXR) signal, or any loop inductance change due 10 a varying plasma position. Thus we have eliminated some other possible explanitions for the loop voltage dips other than a change in conductivity due to electron heating, and possible current drive effects.

For an antenna phasing of $| \pm \pi / 2|$, we measured phase dependent loop voltage drops of $15-25 \%$. The phase change varies the directon of the wave field with respect to

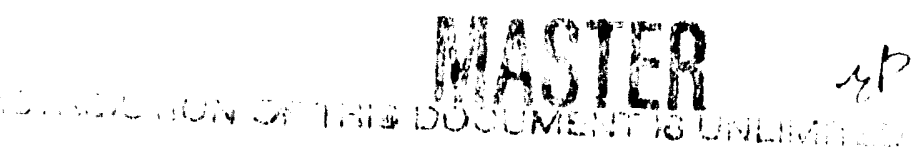


the current (see Figure 1). Our ohmic current feedback system attempts to suppress any change in plasma current due to the RF. We estimate the fractional change in plasma current would be approxiamtely equal to half the fractional change in loop voltage. This yields a value of $7 \%$ of the plasmat current or $5 \mathrm{kA}$ out of $70 \mathrm{kA}$. The average coupled RF power is $2(0) \mathrm{kW}$ for this case at a $\mathrm{B}$ field of $6.7 \mathrm{kG}$ and a line average density of $5 \mathrm{X}$ 10 $12 \mathrm{~cm}^{-3}$.

When the RF power was time modulated on a time scale comparable with the particle confinement time, we observed ion density modulations with the BES diagnostic. In this case the density modulation is presumably due to the increase in particle transport with applied power which is typical in L-mode plasma. However, a detailed understanding of the physics underlying the observed modulation is not required to interpret the data. Two simple assumptions are sufficient: 1) the perturbation, however it is induced, occurs initially at radii where strong power deposition occurs, and 2) this perturbation subsequently propagates through the plasma volume at some finite velocity. The measured phase shift of the BES signal indicated that there were two origins of the power deposition, one at the edge near the antenna and limiter and the other at a minor radius of approximately $6-10 \mathrm{~cm}$ in the core. Figure 2 shows the measured phase shift between the applied RF power and the density modulation measured by BES. Note that clear minima in the phase lag are seen near the limiter radius $(R=117.2 \mathrm{~cm}, \mathrm{r}=24.2 \mathrm{~cm})$ and deeper into the interior $(R=10) \mathrm{cm}, \mathrm{r}=7 \mathrm{~cm})$ which implies two separate regions of strong power deposition at these radii. The observation that the minimum phase lag is about $-\pi$ radians is expected as an increase in RF power degrades confinement and thus leads to a decrease in density and thus the two are 180 degrees out of phase.

Theoretically, we can estimate the location of the mode conversion surface where the phase speed of the launched wave matches the local Alfven speed, and launched wave fields are mode converted to KAW. The cold plasma model and the reduced order full wave code both predict a continuum Alfven resonance in the core of the plasma for our $\mathrm{k}$ spectrum and plasma parameters. This is consistent with the BES data.

\section{New Antenna Designs:}

A new and exciting antenna design, the folded strip line antenna, has been formulated, designed and bench tested and shown to have significantly beneficial characteristics in comparison to a generic strap antenna. A test antenna of this type has been constructed and is currently being installed on the inner wall of one of the port boxes in Phaedrus-T. The folded strip line antenna has a lower inductance than a strap antenna and so a reduced required excitation voltage for a given $B_{d o t}$ in the plasma. This suggests that we can inject significantly more power into this antenna than a strap antenna with the same voltage standoff characteristics. Also, it can be installed next to a vacuum wall without seriously affecting the coupling to the plasma. These features are similar to the folded wave guide designs now of interest for reactor type tokamak devices. However, the folded strip line has a broader bandwidth and can be operated at lower frequencies than the folded wave guide antenna and is thus a better match to Alfven wave heating in Phaedrus-T (and even to Alfven wave heating in a reactor).

\section{Biased H-Mode Plasmas:}

We are presently expanding the variety of plasmas for Alfven Wave experiments in Phaedrus-T. In particular, we have recently started producing biased H-mode plasmas. A typical H-mode behavior (density rise, profile steepening and $D_{\alpha}$ drop) has been achieved in Phacdrus- $T$ in a controlled way by inducing a radial electric field in the plasma edge using a positive bias electrode at $r=23 \mathrm{~cm}$, just inside the limiter. Preliminary data suggest that significant improvement in the particle confinement occurs on the low field side for this bias H-mode case in Phaedrus-T. As we become more 
amiliar with this mode and how it is chanacterized, we will evolve into studying the effects of Alfven Wave current drive and heating in this steep density gradient, H-mode plasma.

\section{The Johns Hopkins MI,M-balsed Soft X-ray Polveliromator Results:}

The Johns Hopkins University Plasma Spectroscopy Group installed the Multilayer Mirror (MLM) based Soft X-ray Polychromator on the Phatedrus-T tokamak at the end of May, 1993. Initially, it was configured to simultaneously measure the Lyman alpha (33.7 angstroms) and Lyman beta (28.5 angstroms) emissions of H-like carbon, along with the Fe XVIII emissions at 16 angstroms and 94 angstroms. On a shot by shot basis the MLM-based polychromator, which has a spatial resolution of $1 \mathrm{~cm}$ and a temporal resolution of $1 \mathrm{~ms}$, was used to scan the vertical axis of the tokamak and measure the emissivity profiles of these charge states. The carbon profiles were measured during a variety of ohmic discharges, including biased $\mathrm{H}$-mode shots, as weil as during the Alfven wave current drive and heating experiments. Ne-like iron was the highest charge state of iron identified in the survey spectra; therefore, no Fe XVIII emission profiles were measured.

During the September tokamak vent, the MLM-based polychromator will be modified to measure the Lyman alpha emission of $\mathrm{H}$-like carbon (33.7 angstroms), the Lyman alpha emission of H-like oxygen (19 angstroms), the singlet and triplet lines of He-like carbon (4().3 angstroms and 40.7 angstroms) and the Fe XVII emission at 15 angstroms. The measured emissivity profiles of these ions coupled with atomic data and the MIST code will be used to calculate the impurity content, transport and radiative losses in the Phaedrus"T tokamak plasma.

\section{Boronization:}

Amorphous boron/carbon films deposited by a de glow discharge of a 0.9 helium and 0.1 trimethlyboron(TMB) gas mixture have been used in the Phaedrus-T tokamak to reduce carbon and oxygen impurities. Auger analysis determined the film composition to have a $\mathrm{B} / \mathrm{C}$ ratio of $1 / 5$. In order to increase the boron content of the film, we developed an in situ molecular oven which sublimates carborane vapor $\left(\mathrm{B}_{10} \mathrm{C}_{2} \mathrm{H}_{12}\right)$ or decaborane vapor $\left(\mathrm{B}_{1}\left(\mathrm{H}_{14}\right)\right.$ into the glow discharge. The film composition of carborane sublimated into a He/TMB glow discharge was measured to be $\mathrm{B} / \mathrm{C} \sim 1 / 1$. We expect to measure even higher values of boron content when sublimating carborane into a pure $\mathrm{He}$ discharge. The film thickness deposited on the vacuum vessel is fairly uniform toroidally, measuring $50 \mathrm{~mm}$ thick at the injection port and decreasing $1040 \mathrm{~mm}$ at a port $160^{\circ}$ away. Carborane is a low toxicity powder at room temperature and is inexpensive and easy to obtain. The higher boron content of the deposited film leads to a reduction of impurity and particle recycling resulting in less $H_{\alpha}$ emission, better plasma density control by means of gats pulfing, and faster machine conditioning after a vent to atmosphere.

\section{Reflectometry:}

In the summer of 1993, we intiated plasma density profile and fluctuation measurements with a simple reflectometer. This reflectometer is in the $8 \mathrm{~mm}$ microwave range and utilizes a used millimeter wave spectrum analyzer and components from interferometer systems previously installed on the Phatedrus-B mirror machine. Fluctuations at the Rl: frepuency have been observed as well ats Mirnov oscillations at low frepuency. ('hanges in the low frepuency fluctuation spectrum have been observed with biased H-mode operatton similar to what is measured with probes. Modifications and improvements to the microwave system are presenty being installed so that we can better determine the location of the Rlifluctuations in the density gradient. 


\section{Edge Probe Experiments:}

Measurements of the plasma edge parameters have been made using triple probes and by monitoring the intensity of the $\mathrm{H}_{\alpha}$ light emitted from the carbon limiter. To date, all the Phaedrus- $T$ antennas have affected $T_{e}$ and $V_{f}$ of probes which are on field lines that connect to the antenna. Since the addition of the boron-nitride (BN) side limiters the increase in $\mathrm{T}_{\mathrm{e}}$ is a few eV and $\mathrm{V}_{\mathrm{f}}$ drops as much as $20 \mathrm{~V}$.

During Alfven wave heating, both with the antenna used in the spring of 1992 and the current two strap antenna, the edge plasma was globally affected. Te and $\mathrm{I}_{\mathrm{sat}}$ increased for all the probes even for the top probe which did not connect to the antenna. $\mathrm{T}_{\mathrm{e}}$ inceased as much as $50 \%$ (from 25 to $40 \mathrm{eV}$ ) for the top probe and the $\mathrm{H}_{\alpha}$ from the carbon limiter doubled. These results are consistent with core heating, which was also suggested the drop in loop voltage and an increase in $T_{\mathrm{e}}(0)$ as measured by Thomson Scattering. Changes in edge parameters were seen even when no drop in loop voltage was observed suggesting that the probes are qualitatively a more sensitive indicator of global rf induced edge modifications.

\section{Up and Coming Experiments:}

Initial studies of low power loading with the test strip line antenna will take place in the next 4 months. High power experiments will be performed after that.

We will continue studies with the two strap antenna to maximize current drive and electron heating and increase the coupled RF power. RF loop probe, BES and reflectometry data will be used to aid in understanding and modeling the flow and absorption of the RF.

Biased $\mathrm{H}$-mode studies will evolve into measurements of RF heating with variable edge density gradient plasmas.

\section{Student Support}

Four Phaedrus students received PhD degrees in the last few months and another is expected to graduate before Nov. 15, 1993. This leaves 11 graduate students associated with Phaedrus. Of these two are expected to finish before June 1994, one is supported by DOD, and one student is on leave and may not return. By eliminating two staff positions and one post doc position we can support 7 graduate students. No offers will be made to new students starting in Sept. 94. I am currently attempting to get additional student support from the university. 
Figure 1. RF on from $100-150 \mathrm{~ms}$. Solid line co-injection $(+\pi / 2)$ (11 discharges averaged); dotted line, counter-injection $(-\pi / 2)$ ( 6 discharges averaged). The loop voltage difference occurs without a marked difference in density. Assuming equal electron heating, the data can be attributed to a drive current of $5 \mathrm{kA}$ out of a total current of 70 $\mathrm{kA}$ at a line averaged density of $5 \times 10^{12} \mathrm{~cm}^{-3}$ 


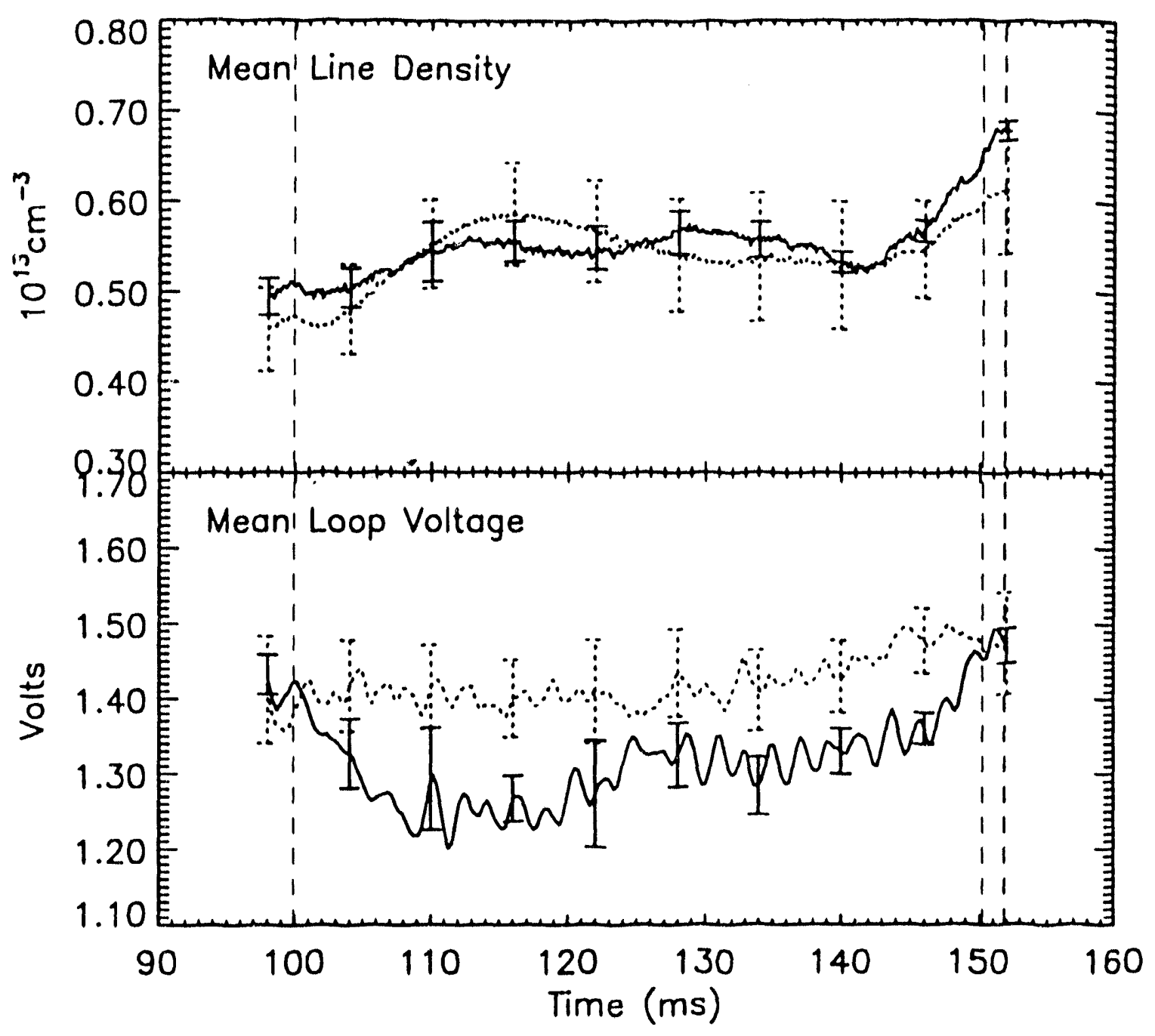

Figure 1. RF on from $100-150 \mathrm{~ms}$. Solid line co-injection $(+\pi / 2)$ (11 discharges averaged); dotted line, counter-injection $(-\pi / 2)$ ( 6 discharges averaged). The loop voltage difference occurs without a marked difference in density. Assuming equal electron heating, the data can be attributed to a drive current of $5 \mathrm{kA}$ out of a total current of 70 $k A$ at a line averaged density of $5 \times 10^{12} \mathrm{~cm}^{-3}$ 


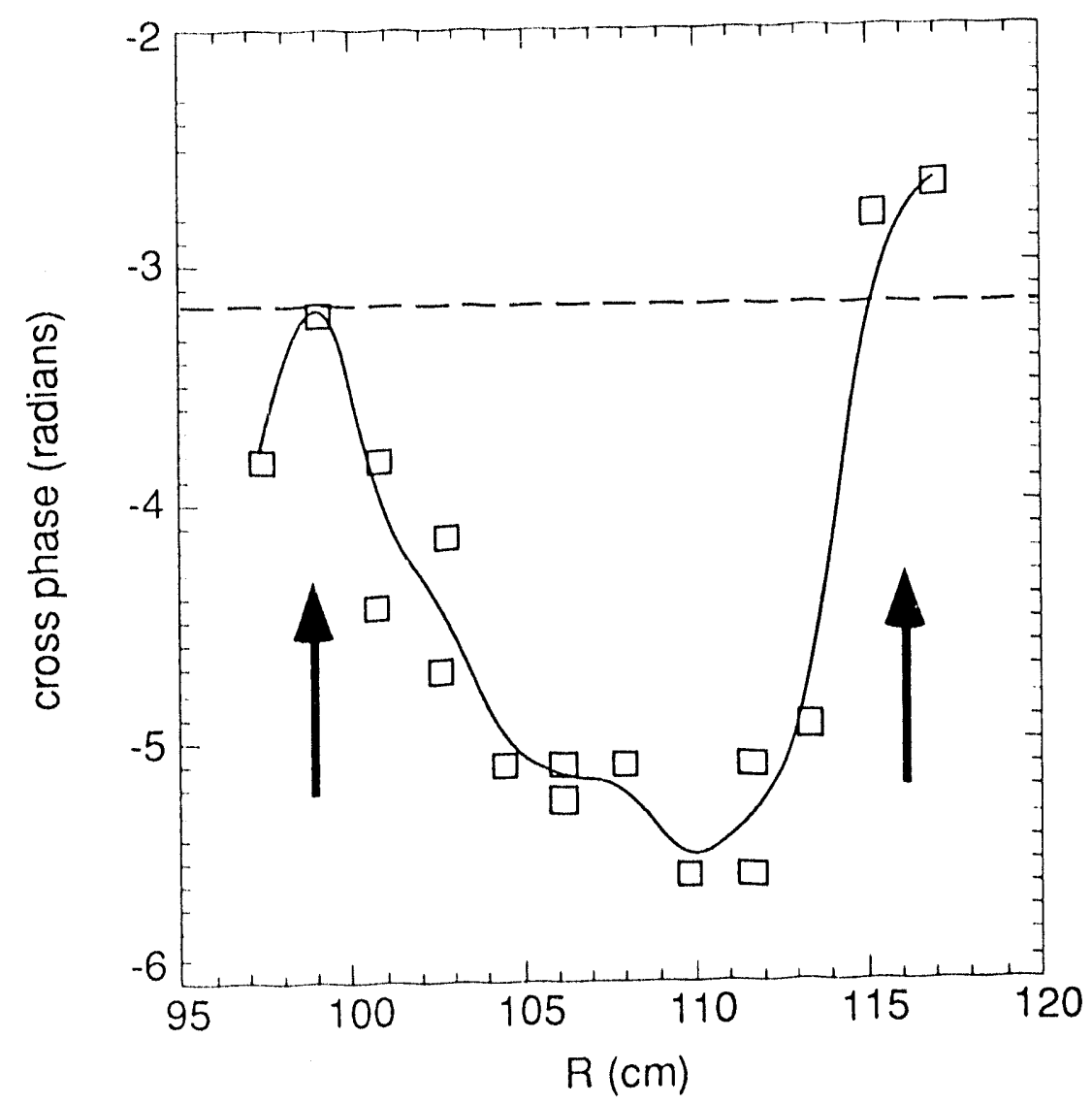

Figure 2. The phase lag between the applied RF power (which was modulated at $500 \mathrm{~Hz}$ ) and the resultant modulation in plasma density (measured via $B E S$ ), $R_{O}=93 \mathrm{~cm}$. 

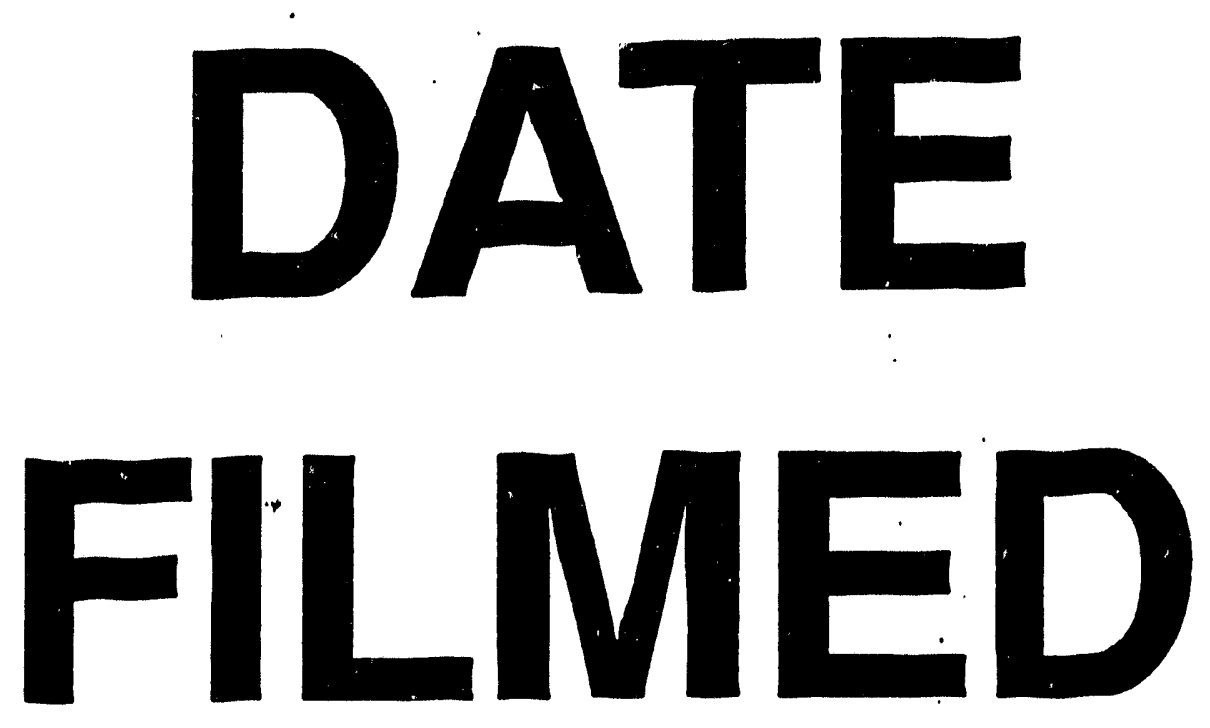

$12 / 13 / 93$
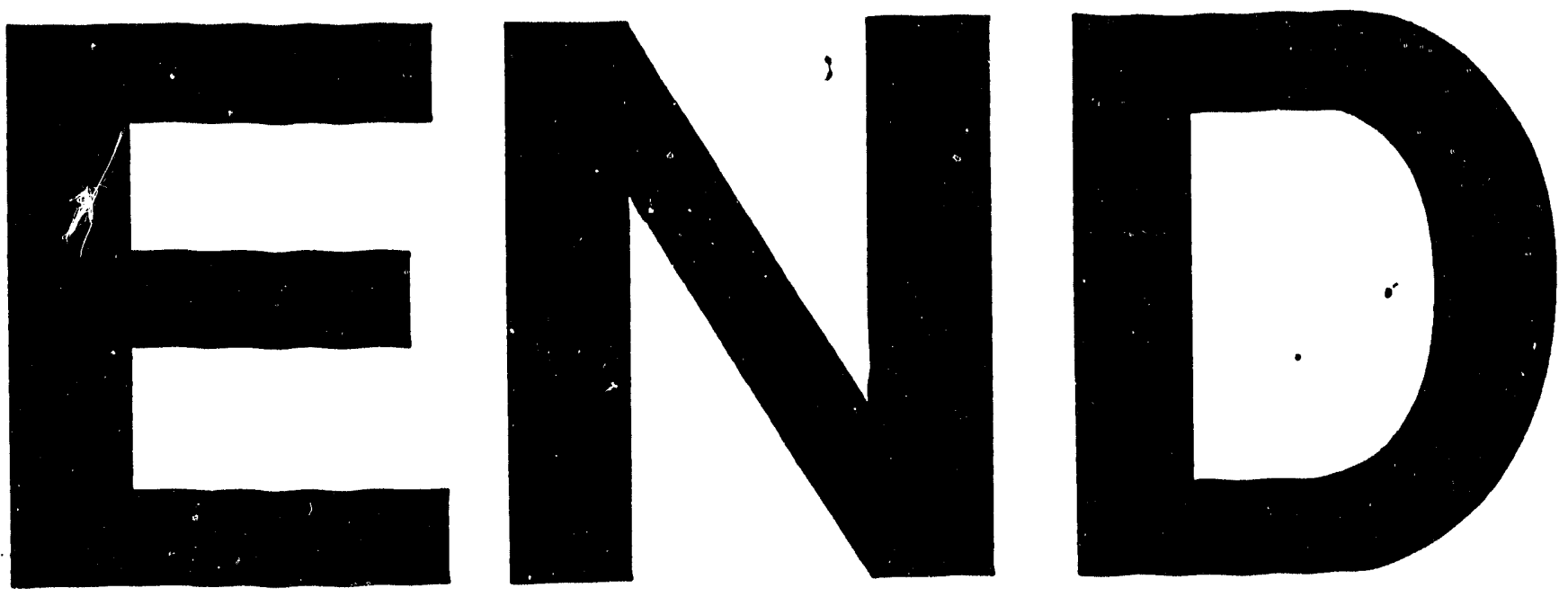

$-\frac{1}{-}-\cdots$ 